\title{
expérimentation en vraie grandeur d'une paroi clouée
}

\author{
projet national clouterre \\ full scale experimental nailed soil retaining \\ french programme : clouterre
}

\section{Résumé}

C. PLUMELLE

C.E.B.T.P."

\begin{abstract}
Dans le cadre du Projet national Clouterre, le C.E.B.T.P. a expérimenté une paroi clouée en vraie grandeur.

L'instrumentation des clous, de la paroi et du sol lui-même a permis de suivre les déplacements de la paroi et du massif cloué, les déformations des clous et leurs efforts en tête pendant les phases d'exécution.

Pour amener la paroi clouée à la rupture, le massif de sable a été saturé. La paroi a péri à la suite d'un glissement d'ensemble d'une partie du massif de sable qui a entraîné des ruptures ou des grandes déformations dans les clous. L'excavation du massif cloué après la rupture a mis en évidence les déformées des clous et du sol.
\end{abstract}

\begin{abstract}
Within the National Project Clouterre, C.E.B.T.P. conducted a full scale experimental nailed soil retaining structure for a vertical excavation in Fontainebleau sand. The nails, the facing and the soil was instrumented to provide the variation of maximum tensile forces with depth at different excavation stages and wall displacements for each stage of construction.

To induce failure, the structure was continuously flooded and the Fontainebleau sand saturated. The structure failed due to excessive facing displacement and large nail strains.

The excavation of the nailed soil retaining structure after its failure focused the nail strains and the soil displacements.
\end{abstract}




\section{PRÉSENTATION DU CONTEXTE DE L'EXPÉRIMENTATION}

Parmi les techniques de renforcement des sols in situ celle concernant le clouage a connu ces dernières années un développement industriel très important; mais l'innovation technologique des entreprises sur les chantiers ayant précédé l'étude du fonctionnement des sols cloués le Projet National CLOUTERRE sur le renforcement des sols par clouage s'est fixé comme objectif final de proposer des Recommandations pour la construction des soutènements cloués provisoires et définitifs.

Dans le cadre de ce Projet National le Centre d'Études et de recherches du Bâtiment et des Travaux Publics (C.E.B.T.P.) a expérimenté une paroi clouée de 7 mètres de haut exécutée par une entreprise spécialisée dans cette technique. Les recherches faites en 1984, 1985 et 1986 ont été financées pour 3/4 par la Fédération Nationale des Travaux Publics et pour $1 / 4$ par la Direction des Affaires Economiques et Internationales du ministère de l'Équipement, du Logement. de l'Aménagement du Territoire et des Transports.

Le but de cette expérimentation a été de réaliser un modèle à l'échelle 1 permettant d'étudier le comportement du massif cloué pendant son exécution et à la rupture.

\section{SITE ET CARACTÉRISTIQUES DE LA PAROI}

L'expérimentation s'est déroulée au C.E.B.T.P. à Saint-Rémy-les-Chevreuse à la Station d'Ouvrages en Terre.

\subsection{Mise en œuvre du massif de sol}

Avant l'exécution de la paroi un massif de sol de $7 \mathrm{~m}$ de haut a été constitué.

Le sable utilisé, du type sable de Fontainebleau, a été mis en place à une densité relative moyenne contrôlée au remplissage.

Simultanément un double polyane a été mis en place sur chaque face latérale du massif central pour assurer une déformation plane lors des essais.

\subsection{Exécution de la paroi}

La paroi clouée a les dimensions indiquées sur les figures 1 et 2 . Le maillage du clouage est de $1.15 \mathrm{~m}$ horizontalement et 1 mètre verticalement.

La paroi a été exécutêe par passes descendantes de 1 mètre. Le choix du type de clou s'est porté sur des tubes en aluminium recuit pour qu'ils puissent reprendre à la fois un effort normal et un moment de flexion tout en gardant une faible résistance à la traction. Leurs longueurs avaient été dimensionnées de telle façon qu'ils ne puissent pas être arrachés pendant l'expérimentation mais qu'ils périssent par cassures ou grandes déformations. Ils ont été scellés dans le sol au coulis de ciment sous basse pression. Chaque tube a été fixé sur le parement en béton projeté d'environ $8 \mathrm{~cm}$ d'épaisseur par l'intermédiaire d'une plaque d'appui boulonnée sur une tige DYWIDAG scellée à l'intérieur du tube.

\subsection{Instrumentation du massif cloué}

Pendant la constitution du massif de sol, on a placé des témoins colorés sous forme de bandes noires horizon-

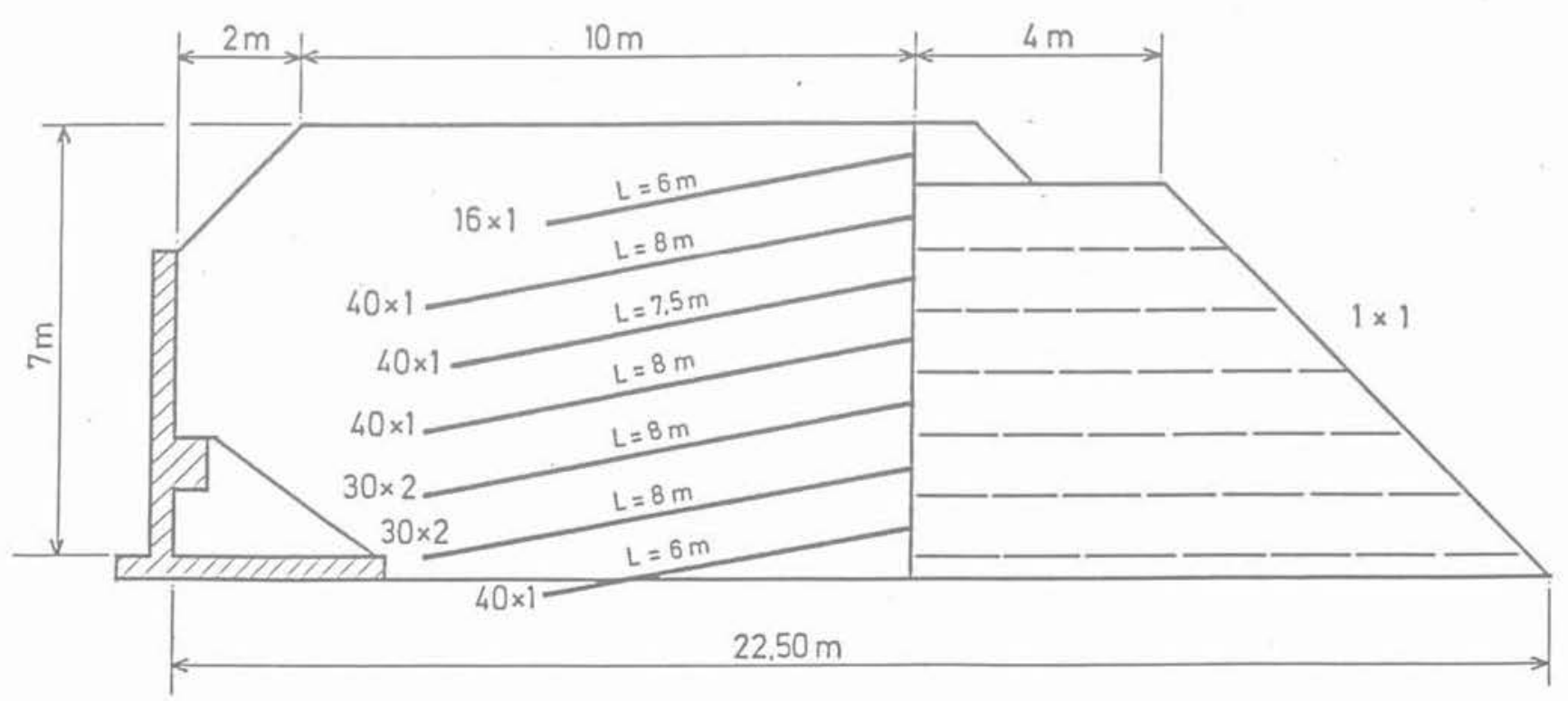

Fig. 1. - Paroi clouée expérimentale.

Fig. 1. - Experimental nailed soil retaining structure. 


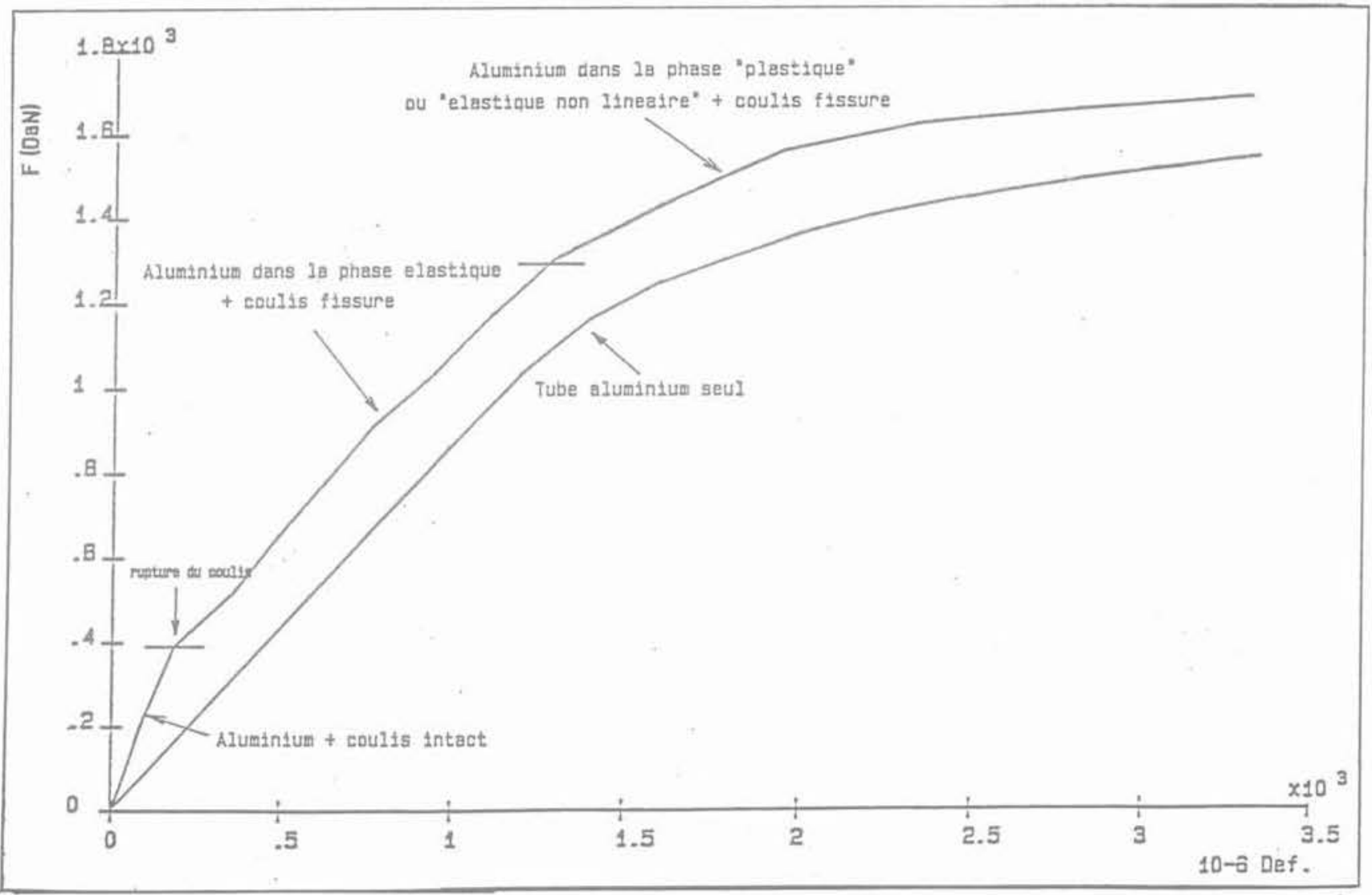

Fig. 4. - Paroi clouée expérimentale : étalonnage des clous en traction.

Fig. 4. - Experimental nailed soil retaining: standardization of nails in extension.

tales perpendiculaires à la paroi tous les mètres au cours du remplissage.

Au cours de l'exécution de la paroi en béton projeté à chaque passe de 1 mètre, trois cibles ont été collées pour suivre les déplacements par micro-triangulation.

Les déplacements à l'intérieur du massif ont été suivis à partir de trois tubes inclinométriques placés à 2,4 et $8 \mathrm{~m}$ de la paroi.

La mesure directe des efforts en tête des clous a été réalisée à l'aide de cales dynamométriques sur les clous centraux des $2^{e}, 3^{e}$ et $4^{e}$ lits. Chaque armature centrale a été équipée de jauges de déformation reliées à deux centrales d'acquisition pilotées par un microordinateur.

\section{OBSERVATIONS DE LA PAROI PENDANT L'EXÉCUTION}

La figure 3 indique les déplacements horizontaux de la paroi et du sol à la fin de l'exécution. On constate une rotation de la paroi par rapport au pied avec un déplacement en tête de l'ordre de $3 \%$. Les déplacements verticaux sont du même ordre que les déplacements horizontaux.
Le passage des déformations aux efforts dans les clous scellés au coulis de ciment est délicat puisque sous un effort donné on peut distinguer jusqu'à trois zones d'efforts : la première où l'aluminium est dans la phase élastique linéaire et le coulis intact, la seconde où l'aluminium est toujours dans la phase élastique linéaire mais le coulis fissuré et la troisième où l'aluminium est dans la phase élastique non linéaire puis dans la phase plastique.

De façon à pouvoir déduire directement les efforts des déformations mesurées, un clou $\varnothing 40 \mathrm{~mm}$ scellé au coulis de ciment dans les mêmes conditions que ceux du chantier a été étalonné. Il a ensuite été déterré pour être mis en traction jusqu'aux grandes déformations.

Sur la figure 4 on distingue bien les trois zones précédemment définies. Pour ce type de clou $\varnothing 40 \mathrm{~mm}$, la rupture du coulis s'est produite à $4 \mathrm{kN}$; on trouve le module de l'aluminium dans la seconde zone, entre 4 et $13 \mathrm{kN}$, soit $66200 \mathrm{MPa}$ pour cet étalonnage alors que l'essai de traction sur le tube d'aluminium a donné un module voisin de $70000 \mathrm{MPa}$.

La distribution des efforts a été tracée à partir d'un programme de dépouillement automatique en prenant les valeurs lissées des déformations, la formule de Ramberg-Osgood $\varepsilon=\frac{\sigma}{E}+0,002 \frac{\sigma}{\sigma(0,2 \%)}$ pour 


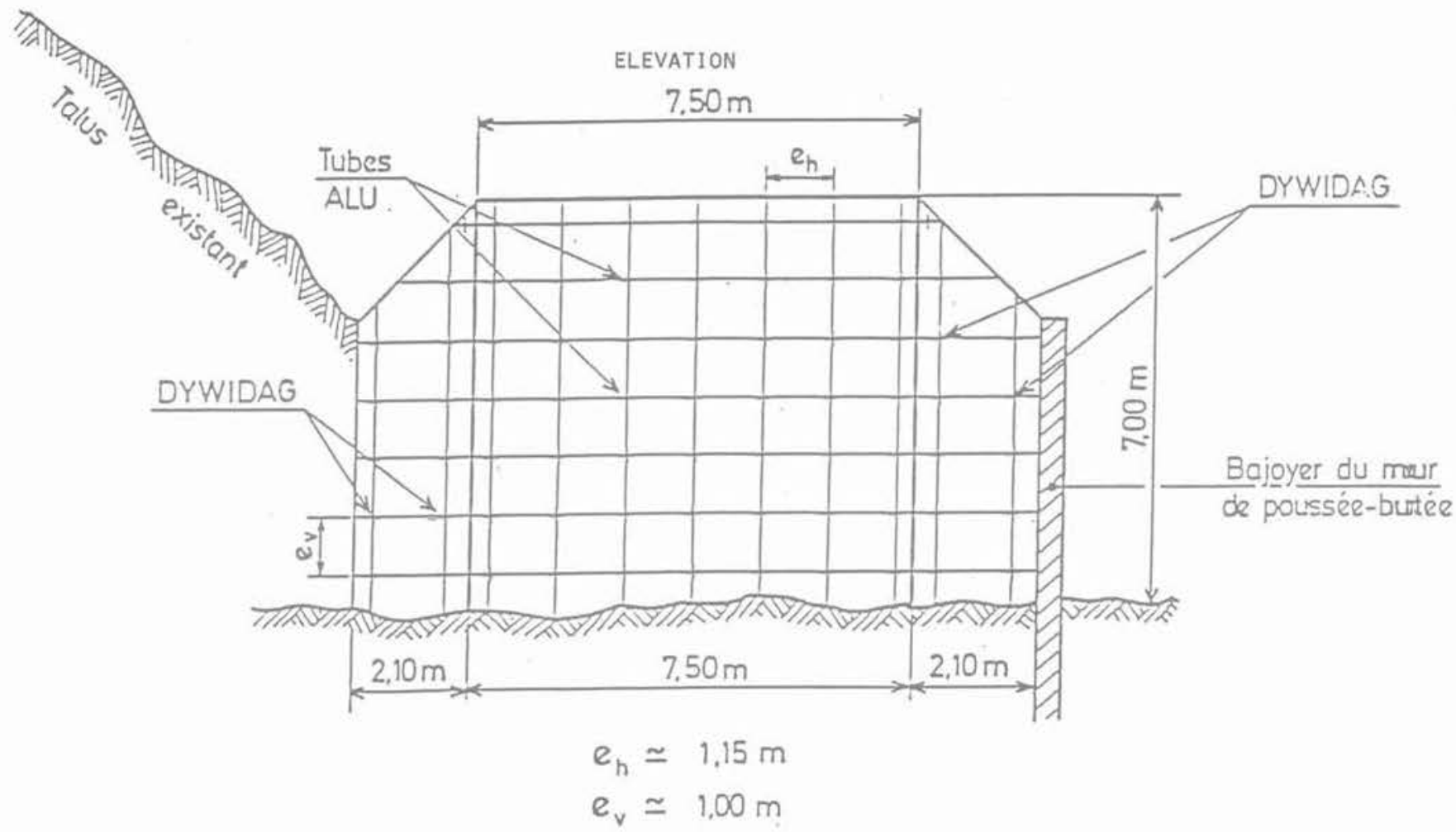

Fig. 2. - Élévation de la paroi clouée expérimentale.

Fig. 2. - Experimental nailed soil retaining structure.

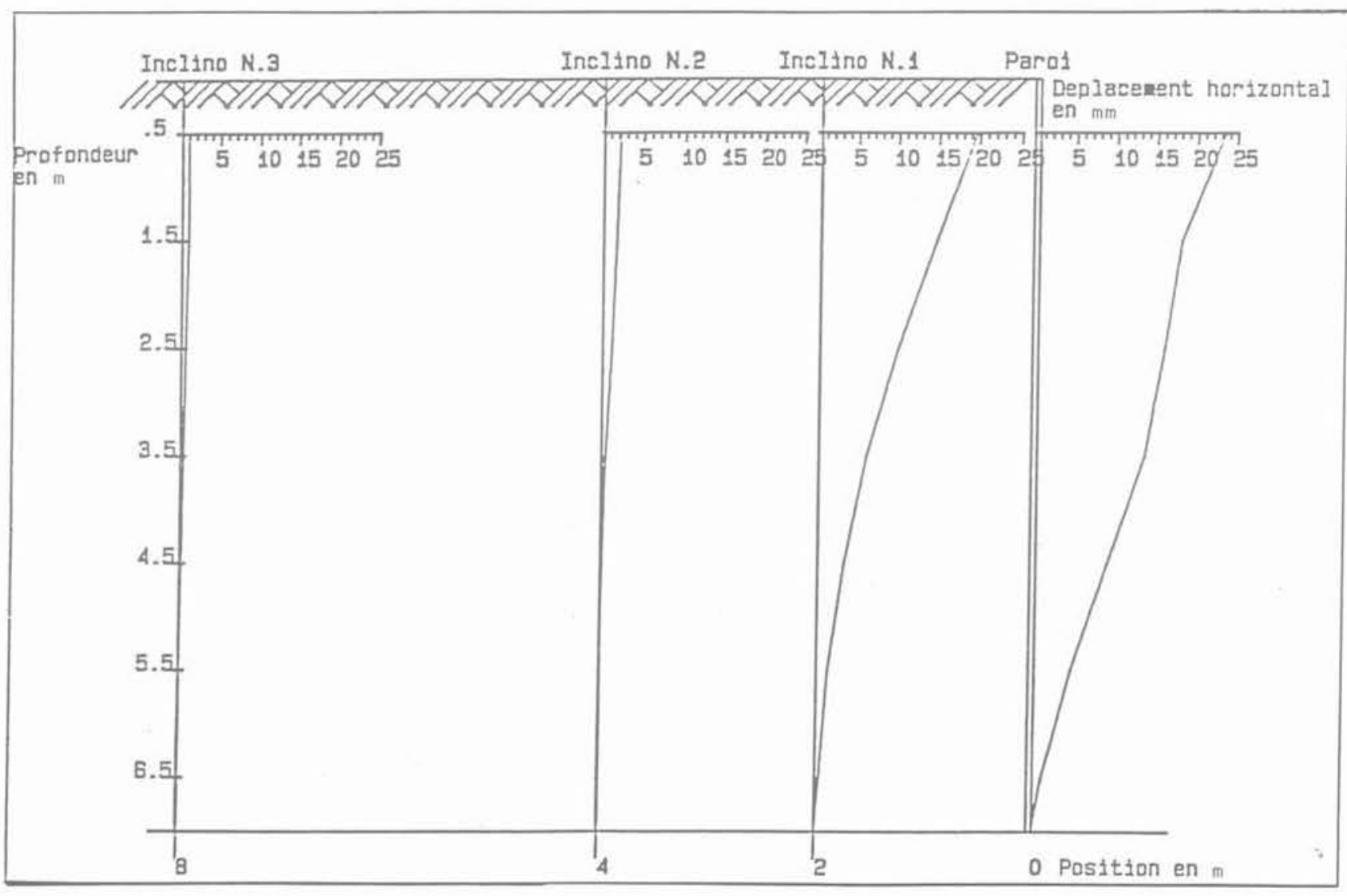

Fig. 3. - Paroi clouée expérimentale : déplacements horizontaux (après fluage).

Fig. 3. - Experimental nailed soil retaining: horizontal displacements (after creep). 


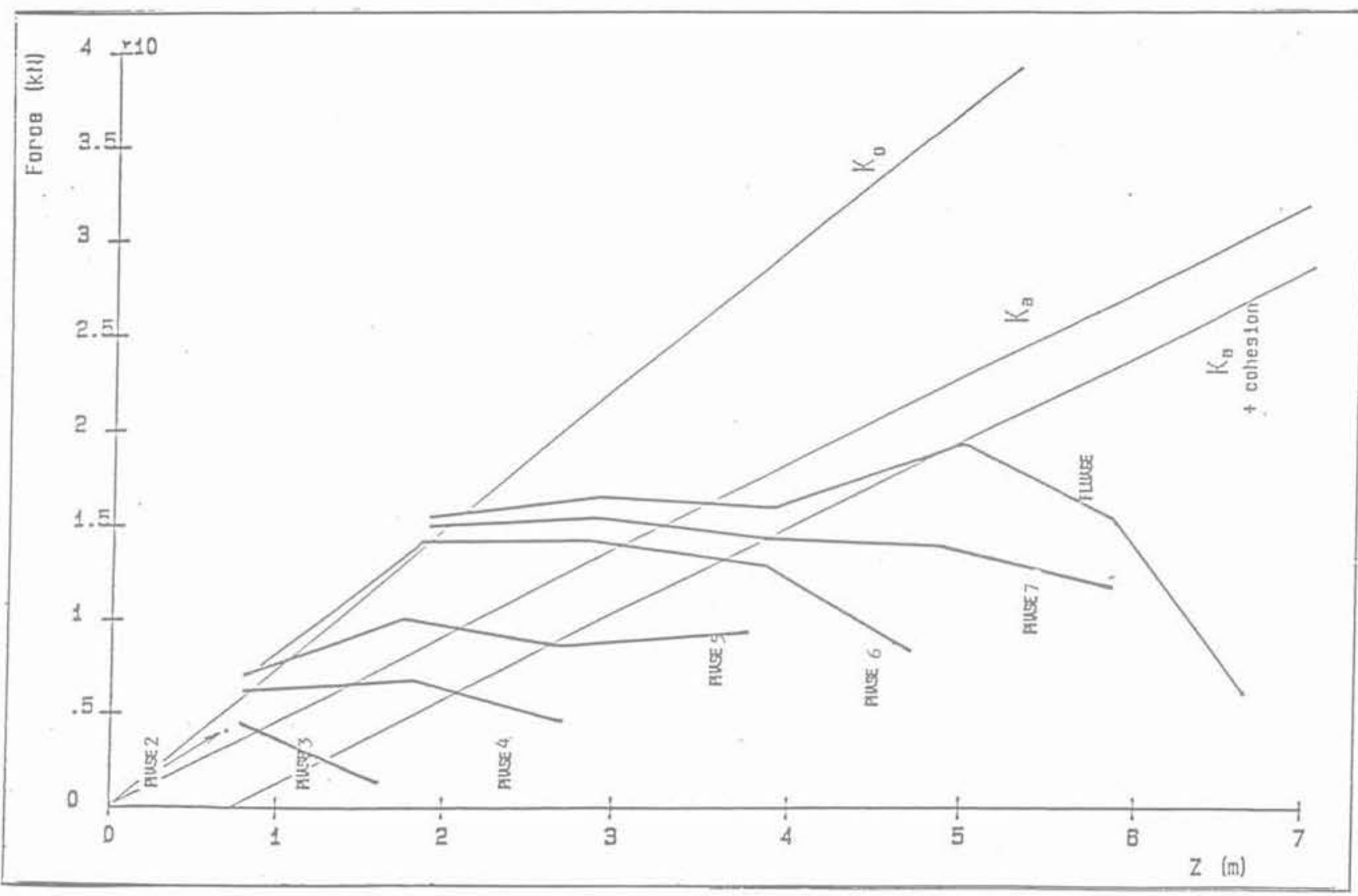

Fig. 5. - Paroi clouée expérimentale : effort maximum dans les barres en fonction de $Z$. Fig. 5. - Experimental nailed soil retaining: maximal strengths in the nails vs depth.

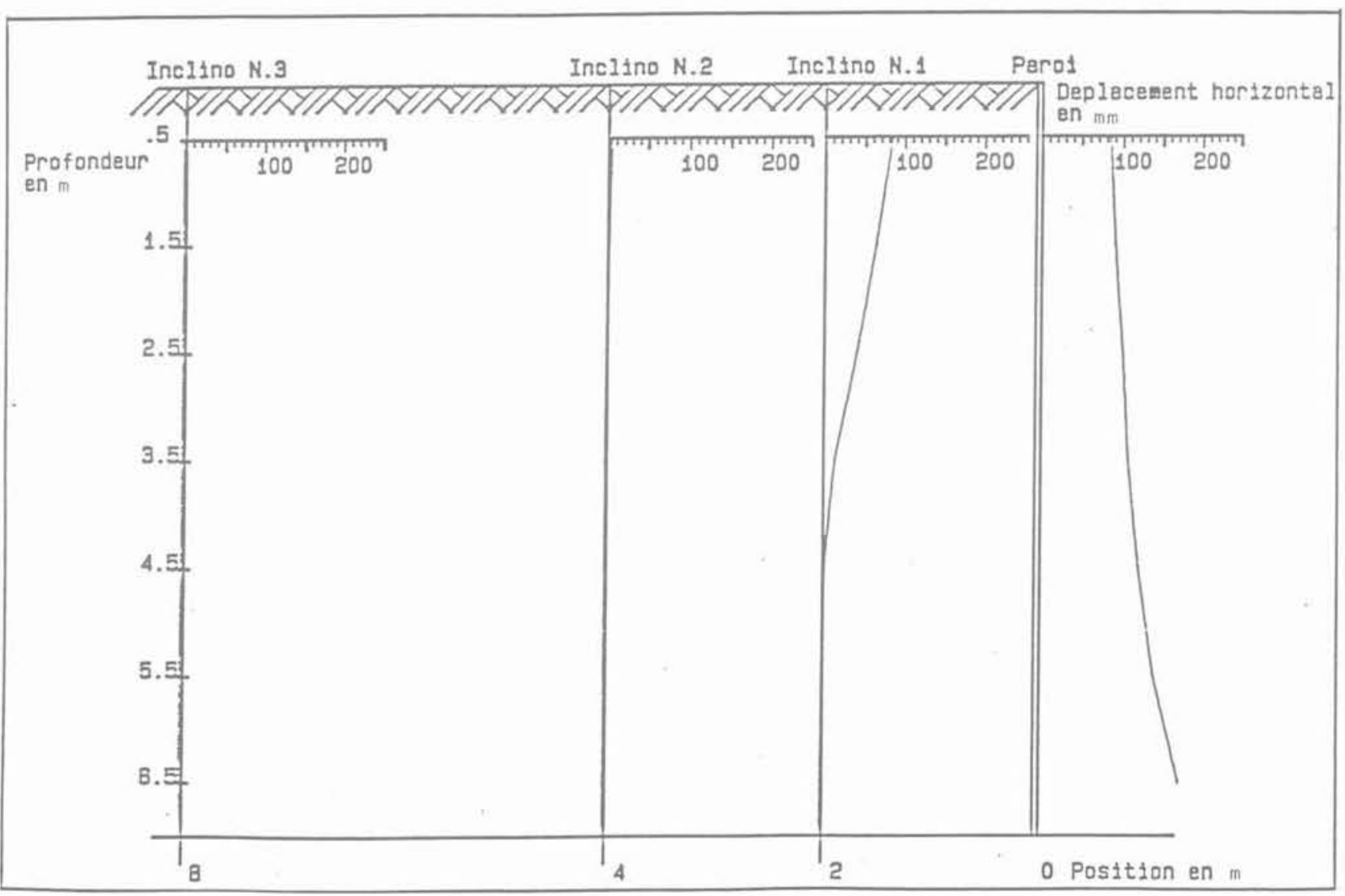

Fig. 6. - Paroi clouée expérimentale : déplacements horizontaux (après rupture).

Fig. 6. - Experimental soil nailed soil retaining structure: horizontal displacements (after breakage failure). 
l'aluminium et en prenant en compte l'étalonnage du clou $\varnothing 40 \mathrm{~mm}$.

la figure 5 montre la répartition des tractions maximales des clous en fonction du phasage ; on l'a comparée à la répartition de la poussée au repos et à la poussée active pour un angle de frottement interne du sable de $\varnothing=38^{\circ}$

L'enregistrement des efforts en tête de clous et des efforts maximaux de traction dans les clous a montré que la mise en charge se fait essentiellement lors des deux phases d'excavation suivant leur scellement.

\section{MISE EN RUPTURE DU MASSIF CLOUÉ}

Trois mois après la fin de l'exécution, le massif de sable cloué a été progressivement saturé pour augmenter son poids volumique et diminuer sa cohésion. Cette méthode présente lavantage de ne pas perturber les champs de déplacements et de contraintes contrairement à celle qui consiste à amener des surcharges en tête de la paroi.

La mise en saturation du sable a amené la rupture de la paroi sans entraîner complètement sa ruine. La paroi s'est affaissée de $27 \mathrm{~cm}$ et s'est encastrée dans le sable sous-jacent, elle s'est avancée de $8 \mathrm{~cm}$ en tête et de $19 \mathrm{~cm}$ en pied.

\section{OBSERVATIONS DU MASSIF CLOUÉ APRESS RUPTURE ET EXCAVATION}

La figure 6 montre à la fois le déplacement de la paroi et du massif après rupture.

La fissure en surface du massif s'est produite à $2.55 \mathrm{~m}$ de la paroi (fig. 7). Le schéma permet de constater

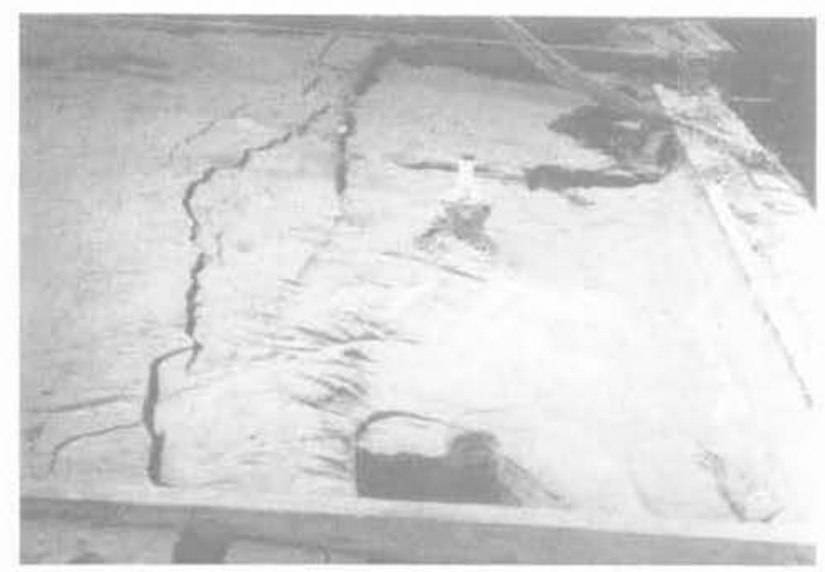

Fig. 7. - Fissures de surface après rupture du massif cloué.

Fig. 7. - Surface cracks after failure of the nailed soil retaining structure. qu'un bloc rigide grossièrement circulaire a glissé lors de la rupture.

L'ensemble des relevés des bandes noires et des déformées des clous pendant l'excavation a permis de reconstituer la totalité de la zone de rupture du massif cloué.

En particulier, on a pu noter que les clous ont subi de grandes déformations de flexion (fig. 8) certains étant mêrne cassés.

La rupture ne s'est pas produite suivant une surface unique mais suivant une zone de largeur variable entre la tête et le pied du massif cloué.

\section{CONCLUSION}

Cette expérimentation en vraie grandeur d'une paroi exécutée par une entreprise mais réalisée en contrôlant les paramètres du sol et en l'instrumentant a permis de suivre son comportement pendant les phases d'exécution et à la rupture.

On a mis en évidence que la cinématique des déplacements est différente lors de l'exécution et à la rupture, rotation par rapport au pied durant les différentes phases de travaux puis glissement pseudo-circulaire d'un bloc rigide à la rupture.

Le lieu des tractions maximales pendant l'exécution est quasi vertical en fonction de la profondeur sauf pour les deux dernières rangées de clous qui ne sont que partiellement mobilisées.

L'ensemble des résultats du rapport complet permettra de caler aussi bien les méthodes de calcul à la rupture que les méthodes de calcul en déplacement.

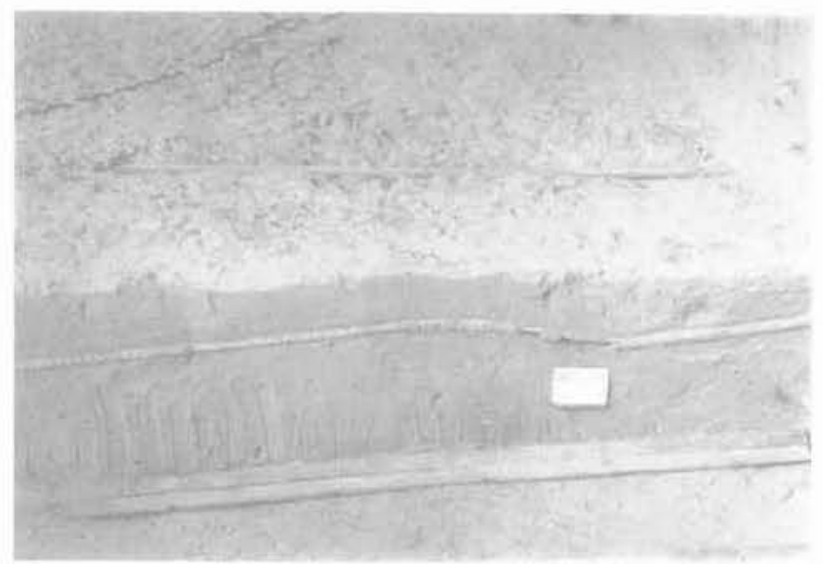

Fig. 8. - Clou déformé après rupture du massif cloué.

Fig. Distorded nail after failure of the nailed soil retaining structure. 